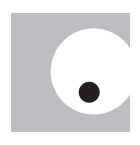

\title{
Los retos más escondidos del cambio climático: Pensamiento global, crisis de comunicación y arte negociadora
}

\author{
Olga Cavallucci de Dalmases*
}

\section{Antecedentes}

E

1 cambio climático no es sólo un fenómeno ${ }^{1}$ de impacto global que afecta de forma trasversal a todos los ámbitos de la vida humana, desde lo económico hasta lo ambiental, pasando por lo político y social, sino que describe un conjunto de elementos que obligan a repensar muchos paradigmas de conocimiento y trasmisión del mismo: en este ensayo trataremos el segundo de estos ámbitos. Indudablemente, la primera respuesta a esta situación se halla en el establecimiento de sistemas económicos que tengan en cuenta los límites naturales del planeta, es decir, de su biocapacidad. Pero esta medida no basta, es indispensable un cambio, una nueva relación del ser humano con su entorno que implique pasar de un enfoque utilitarista a uno integrativo, en donde el hombre ya no domina el medio, sino que se fusiona con este, en una codependencia respetuosa y espontánea, lo cual implica una diferente forma de acercarse al medio, de conocerlo y por ende, de comunicarlo. ${ }^{2}$

Magíster en Relaciones Internacionales, Negociación y Manejo de Conflictos; candidata doctoral en Estudios Latinoamericanos, 2015-2020, Universidad Andina Simón Bolívar Sede Ecuador. Docente investigadora, Universidad Andina Simón Bolívar Sede Ecuador. Docente, Instituto de la Empresa (IDE), Ecuador. Docente, Escuela de la Empresa, Universidad San Francisco de Quito, Ecuador; ‘olgacavalluccid@gmail.com〉.

1. Intergovernmental Panel on Climate Change, Climate Change 2007. Impacts, Adaptation and Vulnerability, (Ginebra: WMO/UNEP, 2007).

2. Carlos Larrea y otros, Post-crecimiento y buen vivir. Propuestas globales para la construcción de sociedades equitativas y sustentable. (Quito: Friedrich-Ebert-Stiftung (FES-ILDIS), 2014). Boaventura de Sousa Santos, Una epistemología del Sur. La reinvención del conocimiento y la emancipación social. (Buenos Aires: Clacso Coediciones, 2009). 
Seguir considerando al crecimiento económico como el pivote central del desarrollo es actualmente un gravísimo error bajo muchos ángulos. Desde el punto de vista ecológico, de manejo de recursos y de los patrones de consumo: el crecimiento económico ha llevado al planeta a una situación de límite, habiendo sobrepasado su capacidad natural de regeneración y de capacidad de adaptación a los cambios climáticos que se han impuesto por la falta de acción política global, lo que en muchos países ya ha causado gravísimas pérdidas humanas y materiales, sobre todo en los más vulnerables desde el punto de vista geográfico y económico. ${ }^{3}$ Y solo se trata del comienzo, si damos crédito al IPCC $\mathrm{y}$ a la comunidad científica internacional. ${ }^{4}$

Ahora bien, si es verdad que en la última década este gravísimo problema global, y el primer problema planetario que la humanidad enfrenta, ha recibido una visibilidad no indiferente comparativamente con las décadas anteriores; por otro lado, los avances en materia de comunicación, en todos los niveles, son profundamente modestos. El cambio climático sigue siendo un tema rezagado que no recibe la atención que debería, teniendo en cuenta que su impacto y consecuencias están poniendo en riesgo nuestra civilización y existencia como especie.

Así, a la dramática ineficiencia de los gobiernos del mundo en liderar acciones locales y a la crisis del multilateralismo -lo que queda evidente en la parálisis de las políticas establecidas en las Conferencia de las Partes del Protocolo de Kioto (COP)- se suman otras dos gravísimas trabas: la ausencia de un componente negociador profesionalizado por parte de países pequeños y extremadamente vulnerables, y la ausencia de una seria política de comunicación global a gran escala, dirigida masivamente a la sociedad civil de todo el mundo, a través de un conjunto de acciones implementadas por instituciones, organizaciones y comunidades, de forma planificada, con capacidad de llegar a todos los ángulos del planeta y toda variedad de audiencia. El precio de no cambiar inmediatamente este rumbo podría ser un colapso civilizatorio, ${ }^{5}$ puesto

3. Carlos Larrea, Hacia una historia ecológica del Ecuador. Propuestas para el debate, (Quito: Universidad Andina Simón Bolívar/Corporación Editora Nacional, 2005).

4. Intergovernmental Panel on Global Change, Cambio Climático 2014 〈https:/ / ipcc.ch/pdf/assessment-report/ar5/syr/AR5_SYR_FINAL_SPM_es.pdf;

5. En este sentido se expresa Lester Brown, presidente del Earth Policy Institute, quien denuncia como las actuales pautas de consumo y hábitos han agotado los recursos del planeta y de no in- 
que, hoy en día, lo que no se comunica, sencillamente no existe. Pero el cambio climático es una realidad que no dejará de profundizarse a pesar de no ser "conocida".

\section{Un primer reto comunicacional: el concepto de "no urgencia intrínseca"}

Una política de comunicación global que pueda definirse como tal, debería estar articulada alrededor de al menos tres ejes principales:

1) Monitorear la vulnerabilidad, adaptación e impactos en personas, bienes y sistemas de producción, en cumplimiento de los compromisos establecidos por la Convención sobre cambio climático (CMNUCC). ${ }^{6}$

2) Sensibilizar y familiarizar a todas las audiencias, en todo nivel de instrucción y edad, acerca de las amenazas existentes para su calidad de vida y calidad de vida de las siguientes generaciones.

3) Educar a las personas de todo nivel socio cultural y edad a la necesidad de un cambio de hábitos, reorientándolos hacia una mayor austeridad y hacia patrones de consumo más éticos y moderados, lo que implica evitar el consumo suntuario excesivo. ${ }^{7}$

A estos se suma una realidad malévola que acompaña a la naturaleza misma del cambio climático: el hecho de que no se lo percibe como una amenaza inminente. Este concepto de "no urgencia intrínseca" podrá ser aclarado mejor con el siguiente ejemplo: si alguien está en su oficina, trabajando como de costumbre, y de repente alguien llama a contarle que su casa está en llamas, seguramente dejará inmediatamente sus tareas y correrá a su casa a la brevedad posible; sin tan siquiera pen-

vertirse este rumbo, apostando a las fuentes de energía renovables, el planeta va irremediablemente hacia un colapso.

6. Convención Marco de las Naciones Unidas sobre el Cambio Climático (1992) ‘http:/ / unfccc.int/ portal_espanol/informacion_basica/la_convencion/items/6196.php .

7. "El consumo suntuario excesivo, -en palabras de Carlos Larrea M.-, se basa en una identificación inadecuada entre la posesión de bienes materiales y la realización personal. Los bienes materiales son un medio, no un fin en sí mismo para la realización de la persona humana. Existen dimensiones de la realización humana que no dependen de la posesión de bienes materiales. A partir de cierto límite, los bienes materiales no necesariamente aportan al enriquecimiento humano, y por contrario, lo pueden distorsionar". En: Carlos Larrea y otros, "Post-crecimiento y buen vivir", 2014. 
sarlo. Lo que ocurre con el cambio climático es muy diferente. El gran público percibe este fenómeno como algo lejano, que afecta los glaciares al otro extremo del mundo y que cuesta la vida de algunas especies animales, como aves o ballenas. Nada más que eso.

Recién a un nivel intermedio de conocimiento, las personas perciben que el cambio climático implica trastornos mucho más complejos gracias a la visibilidad que el fenómeno ha ido adquiriendo en los medios de comunicación especializados. Pero, aun así, la percepción de carácter urgente necesaria tampoco existe en este rango de personas. El cambio climático no es advertido como algo tan grave como perder un empleo, encontrarse con la cuenta corriente en cero o no poder pagar una matrícula escolar para un hijo. Lamentablemente, no hay nada más lejos de la realidad. Según Nicholas Stern, cada año de inacción le cuesta al mundo millones de dólares en términos de PIB mundial; en otras palabras, el tamaño de la riqueza mundial se reduce paulatinamente. ${ }^{8}$

Luego existe un grupo aún más reducido de personas, que llega a tener entre sus manos el Reporte Stern ${ }^{9}$ o algún texto de Lester Brown y llegan a desarrollar una concientización más profunda de lo que es el cambio climático y sobre todo, de lo que está en juego para nuestro futuro como especie.

En resumen, el cambio climático parece ser asunto de pocas élites de técnicos y expertos y no llega a la sociedad civil con el impacto con que debería, arriesgándose a ser poco más que una moda pasajera. Es más, este fenómeno ni siquiera llega a incidir en algunas sociedades puesto que amplios segmentos siguen cuestionando aún su misma existencia $\mathrm{o}$, en el mejor de los casos, priorizando otros temas dentro de su agenda y por ende, dentro de las agendas de los respectivos gobiernos de turno.

Tal es el caso de los Estados Unidos, en donde las preocupaciones principales de las personas están relacionadas con el empleo y la seguridad social, a lo que suman hábitos de consumo y un despilfarro energético absolutamente excesivo y éticamente inaceptable. ${ }^{10}$ Como conse-

8. Nicholas Stern, El Informe Stern: La verdad del cambio climático, (Barcelona: Paidós Ibérica, 2007).

9. Ibíd.

10. Carlos Larrea. "Evidencia científica del cambio climático", clase dictada para la Maestría en Cambio Climático y Negociación Ambiental, Universidad Andina Simón Bolívar, (Quito, año académico 2015-2016). 
cuencia de no haberse manejado una coherente campaña de comunicación a gran escala, eficiente y dramática, no hay el sentido de urgencia ni el conocimiento de cuan grave es este fenómeno. Este inmenso déficit comunicacional acaba generando una situación en la que, por usar una metáfora, el paciente al no saber que está gravemente enfermo no toma las medicinas que necesita, ni implementa las acciones y precauciones apropiadas. Así, a la inacción suicida de los organismos multilaterales y a la inutilidad del Protocolo de Kioto (1997), se suma una apatía generalizada, en donde caminamos hacia un abismo sin sabernos detener. ${ }^{11}$

La comunicación sería una fuerte herramienta de presión política a nivel nacional y multilateral si la opinión pública mundial estuviera alertada por una audaz campaña global de sensibilización y preparación. Las personas se orientarían hacia castigar actividades económicas dañinas para el medioambiente y direccionarían muchas de sus elecciones y consumos hacia pautas más respetuosas con el planeta y sus equilibrios naturales.

Un buen ejemplo de ello es la rígida normativa europea en materia de control de emisiones y de etiquetado verde, a más de una multitud de principios como el de "el que contamina paga" o el de precaución, que castigan comportamientos prejudiciales para el ambiente y al contrario premian a los productos y productores responsables y amigables con el medio ambiente. ${ }^{12}$

Esta conciencia medioambiental no brotó dentro de la Unión Europea de la nada, se trata de una cultura que fue creándose y plasmándose y que ahora está profundamente asimilada dentro del E-28, a todo nivel socio económico. Así, según los "Principios generales y marco bási-

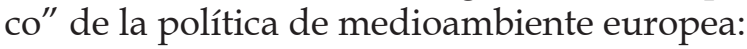

La política medioambiental europea se basa en los principios de cautela, prevención, corrección de la contaminación en su fuente y «quien contamina

11. Los Gases de Efecto Invernadero (GEI/GHG) son aquellos gases que absorben parte de la energía irradiada por la Tierra y la atrapan en la atmósfera. Actúan como una manta que calienta la superficie del planeta. Los principales GEI emitidos por el hombre son el CO2, el Metano, el Óxido Nitroso y gases derivados del flúor. United States Environmental Protection Agency, Climate Change Indicators in the United States, https:/ / www.epa.gov/climate-indicators〉.

12. Parlamento Europeo, La política de medio ambiente: principios generales y marco básico,. 〈http:// www.europarl.europa.eu/atyourservice/es/displayFtu.html?ftuId=FTU_5.4.1.html». 
paga». Los programas de acción plurianuales en materia de medio ambiente fijan el marco de las acciones futuras en todos los ámbitos de la política de medio ambiente. Se integran en estrategias horizontales y se tienen en cuenta en las negociaciones internacionales en materia de medio ambiente. Además, su aplicación es esencial. ${ }^{13}$

Además de su sofisticada y detalladísima normativa, que viene desarrollándose y perfeccionándose desde los años setenta, la UE también ejerce un reconocido liderazgo en materia de negociaciones internacionales sobre el medio ambiente. En este sentido, en la Décima Conferencia de las Partes en el Convenio sobre la diversidad biológica (2010), la UE contribuyó de manera decisiva para la consecución del acuerdo sobre la estrategia mundial para detener la pérdida de biodiversidad en los siguientes diez años. De igual manera, la UE apoyó y sostuvo firmemente la elaboración de los Objetivos de Desarrollo Sostenible globales (ODS) que se dieron a partir de la Conferencia «Río+20» (2012). Finalmente, la Unión ha aportado a la definición del marco normativo en el trascurso de las complejas negociaciones internacionales en la Convención Marco de las Naciones Unidas sobre el Cambio Climático (CMNUCC).

Esta posición firme la llevó a asumir compromisos unilaterales para reducir las emisiones de gases de efecto invernadero, lo que quedó plasmado en la Linking Directive (2008), a través de la cual la UE se comprometía a una reducción del $8 \%$ como bloque frente a la comunidad internacional, sin por ello esperar reciprocidad. Como medidas complementarias, la UE mantiene acuerdos y estrategias de cooperación con diversos países y regiones. En esta línea se ubica, por ejemplo, la Política Europea de Vecindad (países orientales y mediterráneos), a través de la cual se abordan cuestiones extracomunitarias, entre las que se hallan temas medioambientales como la contaminación atmosférica, la calidad del agua, la gestión de residuos, entre otros.

Lamentablemente, el resto de la opinión pública mundial no da señales de un compromiso igual. Los mayores responsables, Estados Unidos y China, siguen silenciosos, presenciando el abuso irresponsable de los recursos de la tierra, sin saber cuan urgente es un cambio drástico

13. Ibíd. 
de patrones de consumo, de matriz energética y finalmente, de modus vivendi. Hasta que estos no decidan emprender un camino similar al de los E-28, la crisis climática persistirá y se agudizará irremediablemente.

\section{La revolución de pensamiento y el reto comunicacional que el cambio climático exige}

La única solución efectiva para resolver la crisis climática es atravesar cambios profundos en el comportamiento y pensamiento humanos. Con el fin de lograr estos cambios es básico el uso de un lenguaje adecuado y simple que vincule la resolución de la crisis con valores compartidos y grandes objetivos. Ha quedado ya demostrado a lo largo de la historia de la humanidad que esta base permite mantener compromisos colectivos en el largo plazo.

Como Aristóteles afirma, somos animales sociales, y nuestra supervivencia a lo largo de los siglos ha estado en gran parte relacionada a la capacidad de cooperar y a la solidaridad entre seres humanos. A esto se suma que los legados intergeneracionales suelen ser acompañados de un sentimiento de responsabilidad hacia las generaciones sucesivas, lo que finalmente contrarresta la tendencia del hombre hacia gratificaciones de corto plazo, egoístas y coyunturales. ${ }^{14}$

Dadas estas premisas conceptuales, uno de los desafíos más importantes que el cambio climático implica es indudablemente el reto comunicacional. Si no sabemos comunicar el peligro que una determinada situación entraña, no solo no estaremos alerta frente a estas posibles amenazas, sino que tampoco podremos presionar para que los tomadores de decisión actúen. Como último eslabón de este efecto dominó, no se darán tampoco esos cambios de patrones de conducta y consumo que van de la mano con la lucha y que suponen trasmitir antes el sentido de urgencia.

Por otro lado, el cambio climático ya ha actuado desde adentro, desencadenando una revolución de pensamiento notable. Sólo para dar un buen ejemplo de ello, tomemos el caso de lo que aconteció en Túnez en

14. Al Gore, Nuestra Elección, Un plan para resolver la crisis climática, (Barcelona: Gedisa, 2009): 311-12. 
2014, país cuyo gobierno insertó dentro de la carta constitucional la lucha al cambio climático, obligando el estado a proteger el planeta para las futuras generaciones, en respuesta a las preocupaciones por la escasez del agua y el incremento de temperaturas, temas particularmente importantes para el área del medio-oriente y norte de África (MENA). ${ }^{15}$

Un enfoque de lo más novedoso viene de un ámbito muy controversial, y desde luego, uno que no suele asociarse con la problemática del cambio climático: el sector financiero. Los inversionistas y todo beneficiario de inversiones vinculados a las instituciones financieras deberán empezar a medir, denunciar y finalmente a reducir los gases de efecto invernadero (GEI/GHG) asociados a estas inversiones, con el fin de no incurrir en problemas regulatorios y fiscales. ${ }^{16}$ Este representa un excelente ejemplo de cómo grupos de presión de la sociedad civil exigen y consiguen que la información relativa a la huella de carbono asociada a esta tipología de actividades económicas sea transparente.

La definición de este tipo de medidas será siempre importante, puesto que la comunidad científica no deja espacio a la duda ni al escepticismo acerca de los impredecibles y exponenciales impactos del cambio climático, lo que finalmente plantea la pregunta de cómo es posible que un sector ligado a intangibles, como lo es el sector financiero, este consiguiendo un logro tan sofisticado mientras que, por otro lado, hasta ahora no se consigue realizar una comunicación efectiva para el cambio climático.

Una primera conclusión es que este tema, al ir en contra de la más poderosa industria del mundo -la petrolera y de combustibles fósiles-, no interesa ser solucionado y mucho menos comunicado, puesto que la idea es que quede relegado a las comunidades científicas de los países y no estalle a nivel global removiendo las fibras sensibles de la sociedad civil.

15. Ed King, "Tunisia embeds climate change in constitution", Climate Home, 27 de enero de 2014. 〈http://www.climatechangenews.com/2014/01/27/tunisia-embeds-climate-change-in-constitution/>.

16. United Nations Environment Programme, UN, Major Investment Firms Call for Increased Focus on Carbon Risk Management and Carbon Accounting in Investment Industry, ‘http:/ / www.unepfi.org/ fileadmin/climatechange/UNEP_FI_Investor_Briefing_Press_Release_-_19_July_2013.pdf). 
Sí, el cambio climático ha ido apuntalándose en la conciencia colectiva global, pero de una forma sesgada y parcializada. Los que más conciencia tienen son los únicos que hacen algo al respecto, demostrando que cuando la comunicación es exitosa, también hay políticas que se materializan a largo plazo. Es de nuevo el caso de la Unión Europea, cuyo esquema de control de emisiones (European Trading Scheme, EU ETS ${ }^{17}$ es legalmente obligatorio para prácticamente la mitad de los operadores económicos vinculados a la industria intensiva de carbono. ${ }^{18}$

Dentro del esquema europeo, los operadores económicos que entran en el grupo de los sectores regulados, del que por ahora quedan excluidos el marítimo, el residencial y algunos otros secundarios, deben, cada año, rendir cuentas de la cantidad de emisiones, que deben corresponder al número exacto de permisos (EUAs). El legislador establece un límite determinando cuanto puede emitir cada país y cada instalación industrial dentro de los mismos, en base a cálculos y estándares histórico-económicos. Por debajo de este límite, los operadores pueden negociar sus derechos a emitir dentro de algunas modalidades previstas, con la idea de que contaminar cueste a quien lo hace y que a la larga, el operador prefiera realizar el cambio hacia tecnologías verdes de última generación, en vez de pagar para conseguir más permisos o de incurrir en multas. ${ }^{19}$

Dejando de lado el mercado de carbono, cuyo funcionamiento y misma razón de ser representan algo controversial por tratarse, a fin de cuentas, de un mercado financiero, con todas las vulnerabilidades que implica. Pensemos que alrededor del $30 \%$ del presupuesto comunitario está dirigido a políticas medio-ambientales, un auténtico lujo si se considera que Europa está atravesando una gravísima crisis financiera, económica y social como consecuencia de la debacle mundial que se desencadenó en septiembre del 2008 y que aún sigue dando importantes

17. Tamara Gilbertson y Oscar Reyes, "Carbon trading. How it Works and why it fails", Critical Currents, No.7, (noviembre 2009).

18. Los llamados traded sectors: cementeras; papeleras, vidrieras, acerías y sector eléctrico entre otros. Estos grupos de empresas a alto consumo energético suma aproximadamente el $47 \%$ de las empresas reglamentadas, es decir, que caen bajo las restricciones y normativas del sistema europeo de control de emisiones, EU ETS. Nota del autor.

19. European Comission, The EU Emissions Trading System (EU ETS), ‘http://ec.europa.eu/clima/ policies/ets_en>. 
coletazos y señales de trasformación profunda, como evidenció la reciente salida del Reino Unido de la Unión Europea.

Todos estos ejemplos, al margen de criterios y opiniones subjetivas, son el resultado de un cambio cultural que a su vez ha sido inducido por apropiadas estrategias comunicadoras masivas, coherentes y efectivas. En resumen, más allá de las probables falencias y desaciertos en el camino, hoy la Unión Europea representa un bloque de países "verdes" que progresan decididamente hacia avances tecnológicos e iniciativas audaces como proyectos de arquitectura eco sustentable, smart grids y planes urbanos regidos por parámetros siempre más estrictos de eficiencia energética. Y es sólo el comienzo...

\section{La comunicación social como estrategia fundamental para la implementación de la CMNUCC}

La comunicación es sin lugar a dudas una herramienta indispensable para implementar la Convención Marco de las Naciones Unidas sobre el Cambio Climático (CMNUUC) y el respectivo mandato que viene de la misma y que establece en su Art. $6{ }^{20}$

Al llevar a la práctica los compromisos a que se refiere el inciso i. del párrafo 1 del artículo 4 de las partes:

a. Promoverán y facilitarán, en el plano nacional y, según proceda, en los planos subregional y regional, de conformidad con las leyes y reglamentos nacionales y según su capacidad respectiva:

1. La elaboración y aplicación de programas de educación y sensibilización del público sobre el cambio climático y sus efectos;

2. El acceso del público a la información sobre el cambio climático y sus efectos;

3. La participación del público en el estudio del cambio climático y sus efectos y en la elaboración de las respuestas adecuadas; y

4. La formación del personal científico, técnico y directivo;

20. United Nations Framework Convention on Climate Change, "Report of the Conference of the Parties on its Eighth Session, held at New Delhi from 23 October to 1 November 2002". New Delhi work programme on Article 6 of the Convention, 23-30, (2003), http:/ / unfccc.int/resource/docs/ cop8/07a01.pdf\#page=23. 
b. Cooperarán, en el plano internacional, y, según proceda, por intermedio de organismos existentes, en las actividades siguientes, y las promoverán:

1. La preparación y el intercambio de material educativo y material destinado a sensibilizar al público sobre cambio climático y sus efectos; $y$

2. La elaboración y aplicación de programas de educación y formación, incluido el fortalecimiento de las instituciones nacionales y el intercambio o la adscripción de personal encargado de formar expertos en esta esfera, en particular para países en desarrollo.

Como puede observarse, la comunicación es un mecanismo fundamental para la gestión del cambio climático debido a que si no se sensibiliza a la población y no se la instruye debidamente acerca del costo político, económico y social de la inacción, la naturaleza jurídica de este instrumento legal que es la Convención se verá seriamente perjudicada en su objetivo de satisfacer exitosamente ciertos objetivos de primaria importancia.

Finalmente, si la Convención fracasará en su propósito de preparar y alertar contra los efectos extremos del cambio climático, así como en el de impulsar la implementación de las acciones y correctivos correspondientes, se condicionarán irremediablemente las posibilidades de que pueda controlarse el fenómeno a escala global y de que puedan menguarse sus consecuencias a la brevedad posible.

Por todas estas razones, y por su misma naturaleza como ciencia de la información, la comunicación se presenta como un eje fundamental y un poderoso recurso social para sostener, canalizar y optimizar la gestión del cambio climático, siendo que, como nos recuerda la famosa oración de James L. Garnett: "Es verdad que la comunicación no es todo, pero es lo único de lo que todo puede depender." ${ }^{21}$ Es así que se necesita que la comunicación sea implementada de una forma racional, es decir, que sea condensada en una arquitectura coherente, según los enunciados de eficaces herramientas como el modelo argumentativo de

21. Pedro Miguel Casado, Analista político/Consultor asuntos públicos, "Estrategia de comunicación de gobierno", presentación de Power Point, (2013), ‘http://www.slideshare.net/ICPiberoamerica/pedro-24311125. 
Stephen Toulmin, que dicta las pautas para una comunicación exitosa; o el Modelo de David Berlo, orientado a guiar la correcta estructuración de un mensaje y el relativo control de audiencia que se consigue al momento de direccionar el mensaje del modo más apropiado.

Entre las potencialidades de la comunicación, cabe destacar la capacidad de fomentar procesos tales como motivación, concienciación, sensibilización, empatía, persuasión y conocimiento, elementos que el padre de la consultoría política moderna, Joseph Napolitano, destaca y subraya debidamente como ejes de una comunicación efectiva. ${ }^{22}$ Por otro lado, la comunicación fomenta el cambio en el sentido más amplio del término: cambios de conducta, de valores, de patrones de consumo, rompiendo el statu quo existente en un determinado entramado social y empujando, por otro lado, profundos reajustes, tanto a nivel de comportamiento individual como a nivel de tendencias dentro de un grupo humano. ${ }^{23}$

Todos estos objetivos no tendrán sentido si no se enfocan en un marco de planificación eficaz, racional y funcional que recoja estas directrices y las plasme en procesos, acciones y estrategias definidas. De continuar así, la comunicación será una plataforma inefectiva, que inclusive podría generar confusión, tanto a nivel de los receptores como a nivel de los emisores de un determinado mensaje, lo que suma a todas las interferencias que existen entre esos dos polos y que a menudo son ignoradas o no sopesadas debidamente. ${ }^{24}$

Si la Convención está principal y formalmente dirigida a ayudar a los países de América Latina en el ámbito de sus compromisos nacionales, en realidad su contenido puede aplicarse a cualquier iniciativa de prevención y protección que se implemente a partir de otras entidades y/o instituciones, así no se trate de procesos liderados por el Estado. La idea es que la comunicación, dentro de este amplio y flexible enfoque,

22. Joseph Napolitan, " 100 cosas que he aprendido en 30 años de trabajo como asesor en campañas electorales" (ponencia, 19 Conferencia Anual de la Asociación Internacional de Asesores Políticos, 1986). https:// drive.google.com/file/d/0B3EKhP0rfNFkLXBfbVhEWVgwRHc/edit».

23. Marco Encalada, Comunicación sobre el cambio climático. Manual para su planificación y práctica en América Latina, (Corporación Oikos/PNUMA, 2005): 8.

24. Las interferencias se refieren a todos esos factores múltiples que distorsionan la calidad del mensaje, en su trayecto entre emisores y receptores: personalidades, contextos, sobreinformación, distracciones, etc. Nota del autor. 
sea un instrumento real, al alcance de todo profesional vinculado a procesos de gestión de cambio climático, es decir, de toda persona que dirija iniciativas vinculadas a la información, al mercadeo y a la sensibilización comunitaria, a la luz de los temas más trascendentes para cada país. ${ }^{25}$ En este sentido, es extremadamente necesario analizar detenidamente los temas evidenciados en las Comunicaciones Nacionales de cada entidad estatal.

Por otro lado, dadas las afinidades geográficas y vulnerabilidades económicas que ureúnen a muchos países de América Latina, la implementación de políticas de adaptación prima sobre las acciones de mitigación; no sólo porque los efectos adversos del cambio climático se hayan mostrado ya, sino porque las emisiones de la región no suman ni el 5\% de las emisiones mundiales. ${ }^{26}$ La paradoja es que los países en vías de desarrollo, cuyo crecimiento es lento y se encuentra aún en fase de consolidación, resultan los más expuestos y afectados a los efectos del cambio climático en la medida en que su capacidad de respuesta es muy inferior respecto a los primeros.

Como se ha destacado, su nivel de emisiones es muy reducido, exceptuando el caso de las grandes economías en desarrollo. ${ }^{27}$ Para temas relacionados a la adaptación, es interesante considerar el Plan de Trabajo de Nairobi (NWP por sus siglas en inglés), ${ }^{28}$ adoptado durante la COP12 en 2006. Así, bajo el Órgano Subsidiario para Asesoramiento Técnico y Tecnológico (SBSTA) de la Convención se ejecuta un programa permanente centrado en impactos del cambio climático, vulnerabilidad y adaptación con el fin de justamente fomentar y dar a conocer estos aspectos, y que las Partes tomen las medidas y acciones apropiadas sobre la base de decisiones informadas, con una base científica y socio económica sólida, tomando en cuenta el cambio climático proyectado y su variabilidad. ${ }^{29}$

25. Ibíd, 9 .

26. Al Gore, Seminario internacional dictado en The Climate Project. (Ciudad de México, Octubre de 2009). También en: Al Gore, Nuestra Elección, Un plan para resolver la crisis climática, (Barcelona: Gedisa, 2009).

27. Se trata de las grandes economías en desarrollo, también conocidas como BRICS. Concretamente: Brasil, Rusia, India, China y Sud África.

28. United Nations Framework Convention on Climate Change, El programa de trabajo de Nairobi, hhttps://unfccc.int/files/adaptation/application/pdf/nwpleaflet_0_es.pdf/.

20. Ibíd. 
Como puede observarse, en el caso de países en vías de desarrollo, el tema comunicacional es particularmente importante, ya que a la criticidad del problema per se, se suma la necesidad de comunicar respuestas rápidas e inmediatas en términos de políticas de adaptación. Por otro lado, como Encalada indica, una comunicación planificada presenta múltiples dimensiones, que identifican desde acciones puntuales, como mini campañas y campañas aisladas de comunicación, hasta la implementación de planes integrales de comunicación, planes estratégicos de mediano y/o largo plazo y finalmente, programas internacionales o regionales de cooperación vinculados a temas comunicacionales de cambio climático.

La tipología de comunicación planificada que se imponga en un determinado contexto nacional dependerá de factores diversos: la criticidad de un problema específico y por ende, la mayor o menor urgencia para comunicarlo a una audiencia; las prioridades que hayan sido identificadas por los responsables del plan de gestión sobre cambio climático; el espacio de maniobra destinado a la comunicación dentro del mencionado plan de gestión versus otros ámbitos de acción; la disponibilidad de recursos económicos y materiales; y finalmente, las capacidades operativas del equipo técnico a cargo de la estrategia de comunicación. Así, la comunicación social integral, tal y como la estamos planteando, es decir como sinónimo de procesos estandarizados y racionales-, constituye un reto complejo y responde al claro objetivo de disciplinar el uso de recursos según un orden marcado por algunas etapas entrelazadas, que además se retroalimentan entre sí: la etapa del conocimiento, la etapa de la decisión y la etapa de la acción. ${ }^{30}$

Este planteamiento sistematizado es indispensable debido a que se trata de un tema que necesita ser transmitido eficazmente a todas las audiencias, con el precedente y al mismo tiempo el desafío, de que que hasta ahora, una campaña de comunicación con este enfoque estratégico y connotaciones no ha sido implementada, concluye Encalada. Es imperativo que la comunicación tenga como objetivo último el de facilitar una transición cultural, social, económica y ecológica. No obstante, este punto de inflexión solo podrá darse a través de un reenfoque de los

30. Marco Encalada, “Comunicación sobre el cambio climático”, 2005. 
canales de comunicación y de la forma de comunicar, lo cual pasa antes por una micro segmentación de las audiencias y por una calibración del mensaje, en función de la tipología de cada audiencia, desde amas de casa hasta gobiernos y organismos multilaterales.

Dicho de otro modo, hay que desmembrar este inmenso público objetivo que es la comunidad internacional y reducirlo a grupos más pequeños, cada uno con sus propias reglas del juego y códigos comunicacionales, como indica el Paradigma de Laswell. ${ }^{31}$ Solo así podrá estructurarse un mensaje con potencial de llegar al público de forma exitosa, del modo más eficaz, con los códigos adecuados y los medios precisos para cierta audiencia y cierto contexto. Solo así el cambio climático aterrizará en la vida diaria de las personas y obtendrá finalmente la relevancia que merece.

\section{La comunicación como herramienta negociadora y la negociación como herramienta de una exitosa comunicación}

Posiblemente, la mejor definición que fija la esencia de la negociación es la que nos viene de los principios enunciados por Sun-Tzu. ${ }^{32}$ "vencer sin luchar, conquistar sin destruir, convertir al enemigo en un aliado."

Dicho de otro modo, la negociación es el arte de llevar al otro de tu lado, persuadiéndolo de tu punto de vista, ganándolo con tus ideas, pero dentro de un escenario en donde no necesariamente se relega a la contraparte en un rol de perdedor. Al contrario, lo deseable es alcanzar un compromiso mutuamente satisfactorio o, en todo caso, aceptable.

Quien es un buen comunicador también termina siendo un excelente negociador, lo que representa un elemento de suma importancia, puesto que las negociaciones finalmente no se dan entre países o instituciones sino entre sus representantes, es decir, entre personas. Así, muchas veces la dificultad de alcanzar acuerdos no reside en la ausencia de potenciales opciones, sino en el fracaso del proceso de comunicación. A

31. "Teoría de comunicación. Información sintética y verídica", Paradigma de Lasswell, ‘https:/ teoriacomunicacion1.wordpress.com/modelos-de-comunicacion/paradigma-de-lasswell/ > .

32. Sun-Tzu, El arte de la guerra, (Buenos Aires: Lonseller, 2004): 16. 
su vez, este proceso depende de varios elementos, comenzando por la forma en la que nos relacionamos con el otro, es decir, por la calidad de nuestra relación con la contraparte. ${ }^{33}$

Las habilidades, destrezas y capacidades que cada uno de estos actores esgrima son extremadamente diversas y de esto dependerán los logros y desempeño de cada país o institución. Sin embargo, el problema de fondo radica en que las personas tienen un diverso trasfondo cultural, lingüístico y axiológico, lo que finalmente repercute en el potencial comunicador si estos elementos no son reconocidos y manejados con sabiduría y experticia.

La comunicación-negociación es parte intrínseca de nuestras vidas, siendo que todos los días realizamos aunque sea "malas negociaciones", en sentido de utilizar esta herramienta sin los fundamentos teóricos que sí existen y sin tener presentes las innumerables interferencias entre emisores y receptores de un mensaje. En este contexto, la tipología de negociación que me propongo considerar es la negociación internacional, es decir, aquella tipología negociadora cuyo objetivo es defender los intereses nacionales, de toda una colectividad, frente al resto del mundo. Y no se trata de tarea fácil, sobre todo cuando hay grandes diferencias en cuanto a la distribución de poder entre estados: es la negociación de la realpolitik, la más agresiva, la más peligrosa, la que entraña las mayores responsabilidades.

Así, el país necesita forjar un equipo negociador, no sólo para que esté preparado a la perfección sobre los puntos de la agenda nacional que se irán a discutir, sino para que sea capaz de saber utilizar todas aquellas poderosas herramientas comunicacionales que nutren al lenguaje y modus operandi del negociador: ambigüedad, sarcasmo, sentido del humor, creatividad, improvisación y las llamadas confidence building measures (CBM). ${ }^{34}$

33. Roger Fisher, Elizabeth. Kopelman y Andrea. Kupfer Schneider, Beyond Machiavelli. Tools for coping with conflict, (New York: Penguin Books, 1996): 5.

34. Gabriella Blum, Islands of Agreement. Managing enduring armed rivalries, (Massachussets and London: Harvard Univeristy Press, 2007): 25. La autora define como "medidas que construyen confianza" cualquier acción, iniciativa que fomente una atmosfera positiva para el dialogo promoviendo la confianza y reduciendo tensiones entre las partes. 
Asimismo, es necesario que pueda sumar a estas habilidades un uso perfecto del idioma inglés, puesto que cualquier mínima diferencia de interpretación será debidamente aprovechada y manipulada por los otros bandos maquiavélicamente..$^{35} \mathrm{~A}$ más de estos ingredientes, hay que considerar otro tema de fondo: la asimetría de poder y de recursos que suele existir entre las partes. Tomemos el caso de una delegación de cambio climático en donde se enfrenten países del primer mundo y países en vías de desarrollo: los delegados de países en desarrollo deben saber oponerse a determinadas medidas, ya sea creando coaliciones y bloques con países de intereses afines o bien con estrategias negociadoras orientadas a añadir o retirar un punto de los textos sin crear conflictos muy evidentes. ${ }^{36}$

A más de esto, las delegaciones de países en desarrollo suelen presentarse en condiciones numéricas muy inferiores y con equipos adversarios que además de su impacto físico, cuentan con profesionales de la negociación que manejan el mismo tema desde hace décadas, perfectamente bilingües y que no han rotado en cambios de gobierno, por lo que conocen a la perfección las reglas del juego, las técnicas, los trucos, y por supuesto, los temas que se van a discutir, por haberlos manejado en múltiples ocasiones. Como puede verse, conocimiento técnico, conocimiento de la agenda nacional y de los temas a tratar estan lejos de ser elementos suficientes, aún si estos requisitos fueran garantizados y cubiertos por hábiles negociadores.

Es evidente que se necesita mucha más preparación, tiempo para revisar textos de negociación desde un punto de vista crítico y filosóficamente analítico ${ }^{37}$ tiempo para analizar el historial de cada negociación desde un inicio: decisiones anteriores, accidentes, incidentes, jurisprudencia, etc. A la vista de este breve análisis cabe preguntarse cuáles de estos factores podemos decir tener asegurados desde nuestra perspectiva país, siendo que el Ecuador es parte de de muchos procesos multilaterales y que finalmente, presenta todas las vulnerabilidades de un pequeño país en de desarrollo. La discusión es amplia, presenta muchas

35. Andrés Mogro, Las Negociaciones internacionales en cambio climático y los países en desarrollo ¿Qué hacer? (tesis de posgrado, Universidad Andina Simón Bolívar, Sede Ecuador, 2013).

36. Ibíd.

37. Ibíd. 
aristas y podría inclusive ser políticamente controversial. No es la idea debatir este punto en este espacio. El objetivo, por lo pronto, es sólo el de dar una señal de alarma, puesto que todos queremos lo mismo, más allá de ideologías y posiciones: el progreso económico, social y cultural y el respeto por parte del resto de la comunidad internacional.

\section{Conclusiones: el científico comunicador como nueva propuesta comunicacional}

A la luz de este breve análisis acerca de estas falencias comunicacionales con respecto al cambio climático, es evidente como desde una perspectiva académica y sociológica, sea indispensable crear un puente entre ciencia y comunicación, entendiendo como la segunda la capacidad de "llegar" exitosamente con este mensaje de urgencia a todo tipo de público. Sin esta banda transmisora, de nada sirve el conocimiento y toda ciencia acaba muriendo entre estériles debates académicos que no salen de unas pocas aulas y que no interesan sino a limitadas élites y grupos de personas.

La complejidad del cambio climático requiere un serio trabajo en el sentido de evitar caer en una filosofía de moda, en un momento pasajero. Al contrario, se va a requerir una especie de gobierno de coalición mundial, enmarcado en un estado de emergencia global, en donde cada país adopte una nueva lógica de ahorro energético, de austeridad, de no-consumismo, dentro de un marco en donde cada uno tiene un rol y puede contribuir en algo. Como Larrea indica, no se trata únicamente de una escasez de recursos per se: se trata sobre todo de un tema ético, puesto que, contrariamente a lo que afirman los neomalthusianos y los neoclásicos, el planeta sí tiene capacidad para sustentar a todos sus habitantes ${ }^{38}$ el problema es que hay una extraordinaria inequidad a la hora de cómo estos recursos están distribuidos.

Así, a la vista de estos dramáticos desafíos, ciencia y comunicación deben hacer una alianza estratégica apuntando a que entiendan la en-

38. Fernando Estenssoro Saavedra, Historia del debate ambiental en la política mundial 1945-1992. La perspectiva latinoamericana, (Santiago de Chile: Instituto de Estudios Avanzados, Universidad Santiago de Chile, 2014): 66-71. 
vergadura del problema, su transversalidad, su impredecibilidad y sobretodo, conozcan los riesgos que la inacción representa para nuestra sociedad y para el planeta entero. Esta alianza constituirá la verdadera diferencia entre una improductiva discusión académica y una auténtica y potencial herramienta de lucha al cambio climático, en donde el científico sea comunicador y el comunicador sea científico.

En definitiva, con respecto al cambio climático, es evidente como, a parte de mejorar los niveles de conciencia y conocimiento generales, y sus impactos negativos en la economía, salud, sociedad, biodiversidad, etc., una comunicación efectiva y bien planificada llevará a la población a un cambio de patrones de conducta y hábitos, lo que nos hará más resistentes y adaptables; según muchos, las únicas opciones que nos quedan. ${ }^{39}$ Este objetivo responde a una necesidad global, puesto que la humanidad entera debe aspirar a economías pos-petroleras, basadas en energías renovables, políticas de eficiencia energética y ahorro de recursos, ya que, si le damos crédito a la teoría del Pico de Hubbert, los recursos se están agotando para todos, mundo desarrollado y mundo en desarrollo.

Por ello, por esa ambición de fondo, una instancia básica dentro de cualquier estrategia de comunicación en apoyo a la gestión sobre el cambio climático será, obviamente, determinar a dónde se quiere llegar, cuáles son los objetivos, cómo queremos alcanzarlos y en qué lapso de tiempo. En otras palabras, se trata de identificar metas de comunicación bien definidas y de insertarlas en un cronograma de trabajo preciso y estricto, sabiendo que el tiempo no es nuestro aliado, sino todo lo contrario.

Pero esto tampoco basta: la idea de fondo consiste en buscar un marco multilateral más incluyente, que apunte a involucrar todo tipo de actores, desde ciudades, compañías, gobiernos, corporaciones, hasta los citadinos comunes, en un esfuerzo colectivo global, puesto que el cambio climático, al igual que otros problemas globales, requiere de un compromiso universal ponderado a las especificidades, fortalezas y debilidades de cada contexto nacional. Como puede observarse, la agen-

39. Federico Rampini, Banchieri,(Milano:Mondadori, 2013): 157-162. 
da es tremendamente audaz y solo podrá lograrse en la medida en que se sensibilicen audiencias siempre más amplias a la gravedad del problema. Por otro lado, un público más preparado, sensible y consciente ejercerá muchas más presiones sobre los tomadores de decisiones, lo que finalmente generará un círculo virtuoso hacia la implementación de políticas efectivas y apropiadas. Sólo de este modo podrá contrastarse el enorme poderío y lobbying de los sectores vinculados al business as usual, es decir a la mayor industria del mundo, la del carbón fósil: petróleo, carbón y gas. ${ }^{41}$

La comunicación, al igual que la negociación, son herramientas poderosas, pero curiosamente, ambas comparten el triste destino de ser subestimadas en su potencial como notables factores de cambio. Es hora de romper este patrón. Es hora de potenciar y fortalecer ambas, puesto que ninguna buena idea es tal, si no logra ser comunicada a sus destinatarios o negociada hábilmente, dependiendo de los casos. La ventana de oportunidad que el cambio climático nos concede es mucho más pequeña de lo que puede parecer, el tiempo corre y no podemos darnos el lujo de ignorar ninguna arma que tengamos a nuestra disposición. Saber comunicar y saber negociar el cambio climático se ha convertido en una cuestión de sobrevivencia.

\section{Bibliografía}

Blum, Gabriella. Islands of Agreement. Managing enduring armed rivalries. Massachussets and London: Harvard Univeristy Press, 2007.

Brown, Lesser. Plan B 4.0: Mobilizing to save civilization. Nueva York: Norton Books, 2009. IPCC. Cambio climático 2007, Informe de síntesis. OMM., en: 〈http:/ /www.ipcc.ch/pdf/ assessment report/ar4/syr/ar4_syr_sp.pdf).

Intergovernmental Panel on Global Change. Cambio Climático 2014, ‘https://ipcc.ch/ pdf/assessment-report/ar5/syr/AR5_SYR_FINAL_SPM_es.pdf>

Casado, Pedro Miguel. Analista político/Consultor asuntos públicos. "Estrategia de comunicación de gobierno". Presentación de Power Point (2013). «http:/ / www.slideshare.net/ICPiberoamerica/pedro-24311125.

41. Richard Heinberg, Searching for a Miracle. "Net energy" limits and the fate of industrial society. A joint Project of the International Forum on Globalization and Post Carbon Institute, (San Francisco: Chroma Graphics, 2009): 3. 
Depledge, Joanna y Farhana Yamin. The International Climate Change Regime, A Guide to Rules, Institutions and Procedures. (Nueva York: Cambridge University Press, 2004).

La Via Eco. "El poder de la información: el art. 6 de la CMNUCC". La Via Eco, 20 de enero de 2011. 〈https://laviaeco.wordpress.com/2011/01/20/el-poder-de-la-informacion-el-articulo-6-de-la-cmnucc/s.

Encalada, Marco A. Comunicación sobre el cambio climático. Manual para su planificación y práctica en América Latina. (Corporación Oikos/PNUMA, 2005).

Estenssoro Saavedra, Fernando. Historia del debate ambiental en la política mundial 19451992. La perspectiva latinoamericana. (Santiago de Chile: Instituto de Estudios Avanzados. Universidad Santiago de Chile, 2014).

European Comission. The EU Emissions Trading System (EU ETS). «http://ec.europa.eu/ clima/policies/ets_en.

Fisher, Roger, Elizabeth. Kopelman y Andrea Kupfer Schneider. Beyond Machiavelli. Tools for coping with conflict. (New York: Penguin Books, 1996).

Gilbertson, Tamara y Oscar Reyes. "Carbon trading. How it Works and why it fails”. Critical Currents, No. 7, (2009).

Gore, Al. Nuestra Elección, Un plan para resolver la crisis climática. Barcelona: Gedisa, 2009. Seminario internacional dictado en The Climate Project. (Ciudad de México, Octubre de 2009).

Grupo Intergubernamental de Expertos sobre el Cambio Climático. Cambio climático 2007, Informe de sintesis. (Ginebra: OMM, 2008). En: ‘http://www.ipcc.ch/pdf/assessment-report/ar4/syr/ar4_syr_sp.pdf >

Heinberg, Richard. Searching for a Miracle. "Net energy" limits and the fate of industrial society. A joint Project of the International Forum on Globalization and Post Carbon Institute. San Francisco: Chroma Graphics, 2009.

Intergovernmental Panel on Climate Change., Climate Change 2007., Impacts, Adaptation and Vulnerability. Ginebra: WMO/, UNEP, 2007.

King, editor. "Tunisia embeds climate change in constitution". Climate Home, 27 de enero de 2014. «ttp:/ / www.climatechangenews.com/2014/01/27/tunisia-embeds-climate-change-in-constitution/s.

Larrea, Carlos. "Evidencia científica del cambio climático". Clase dictada para la Maestría en Cambio Climático y Negociación Ambiental, Universidad Andina Simón Bolívar (Quito, año académico 2015-2016).

-----. Hacia una historia ecológica del Ecuador. Propuestas para el debate. (Quito: Universidad Andina Simón Bolívar/Corporación Editora Nacional, 2005).

Larrea, Carlos y otros. Post-crecimiento y buen vivir. Propuestas globales para la construcción de sociedades equitativas y sustentables. (Quito:Ecuador, Friedrich-Ebert-Stiftung [FES-ILDIS], 2014).

Mogro, Andrés. Las Negociaciones internacionales en cambio climático y los países en desarrollo ¿Qué hacer?. Tesis de posgrado, Universidad Andina Simón Bolívar, Sede Ecuador, Quito, 2013.

Napolitan, Joseph. "100 cosas que he aprendido en 30 años de trabajo como asesor en campañas electorales". Ponencia presentada en la 19 Conferencia Anual de la 
Asociación Internacional de Asesores Políticos, 1986. «https:/ / drive.google.com/ file/d/0B3EKhP0rfNFkLXBfbVhEWVgwRHc/edit>.

Organización de las Naciones Unidas. Convención Marco de las Naciones Unidas sobre el Cambio Climático (1992) 〈http://unfccc.int/portal_espanol/informacion_basica/ la_convencion/items/6196.php>.

Ohlinger, Tina. La política de medio ambiente: principios generales y marco básico. (2008). 〈http:// www.europarl.europa.eu/atyourservice/es/displayFtu.html?ftuId=FTU_5.4.1.html.

Rampini, Federico. Banchieri. (Milano: Mondadori, 2013).

Sousa Santos, Boaventura. Una epistemología del Sur. La reinvención del conocimiento y la emancipación social. México-Argentina Buenos Aires: Clacso Coediciones, 2009).

Stern, Nicholas. El Informe Stern: La verdad del cambio climático. (Barcelona: Paidós Ibérica, , 2007).

Sun-Tzu. El arte de la guerra. (Buenos Aires: Lonseller, 2004).

United Nations Environment Programme. UN, Major Investment Firms Call for Increased Focus on Carbon Risk Management and Carbon Accounting in Investment Industry. 〈http://www.unepfi.org/fileadmin/climatechange/UNEP_FI_Investor_Briefing_Press_Release_-_19_July_2013.pdf>.

United Nations Framework Convention on Climate Change. El programa de trabajo de Nairobi. 〈https://unfccc.int/files/adaptation/application/pdf/nwpleaflet_0_ es.pdf>.

United Nations Framework Convention on Climate Change. "Report of the Conference of the Parties on its Eighth Session, held at New Delhi from 23 October to 1 November 2002". New Delhi work programme on Article 6 of the Convention, 23-30. (2003). 〈http:/ / unfccc.int/resource/docs/cop8/07a01.pdf\#page=23〉.

United States Environmental Protection Agency. Climate Change Indicators in the United States. 〈https:/ / www.epa.gov/climate-indicators〉. 